\title{
The relation between significant stenosis or dilatation at the repair site and outcome in a contemporary cohort of patients with repaired aortic coarctation as assessed using Cardiovascular Magnetic Resonance
}

\author{
Sylvia S Chen ${ }^{1 *}$, Konstantinos Dimopoulos ${ }^{1,2}$, Rafael Alonso-Gonzalez ${ }^{1}$, Emmanouil Liodakis ${ }^{1}$, \\ Elvis Teijeira-Fernandez ${ }^{1}$, Maria Alvarez-Barredo ${ }^{1}$, Gerhard-Paul Diller ${ }^{1,2}$, Daryl Shore ${ }^{1}$, Anselm Uebing ${ }^{1}$, Lorna Swan ${ }^{1}$ \\ , Michael Gatzoulis ${ }^{1,2}$, Raad H Mohiaddin ${ }^{1,2}$ \\ From 15th Annual SCMR Scientific Sessions \\ Orlando, FL, USA. 2-5 February 2012
}

\section{Summary}

Complications of restenosis or dilatation at the site of repair may occur late after initial repair of coarctation of the aorta $(\mathrm{CoA})$. Cardiovascular magnetic resonance (CMR) is becoming the choice of imaging of patients after CoA. Using CMR, we studied the relation between these complications and outcome inn patients with previous CoA repair, and found that significant stenosis or dilatation at the repair site is a predictor of adverse outcome.

\section{Background}

Cardiovascular magnetic resonance (CMR) is becoming the method of choice for assessing repaired CoA patients, especially for identifying important long-term sequelae of restenosis or dilatation at the repair site. We examined the prevalence of restenosis and dilatation at the site of aortic coarctation $(\mathrm{CoA})$ repair and their relation to long-term outcome in patients with repaired CoA.

\section{Methods}

Data and imaging for adult CoA patients followed in our institution were analysed. CMR images of the aorta were studied, and the diameter of the aorta at the repair site was measured on CMR and its ratio to the aortic

${ }^{1}$ CMR Unit, Royal Brompton Hospital, London, UK

Full list of author information is available at the end of the article diameter at the diaphragm (repair site-diaphragm ratio, RDR) calculated. Significant restenosis was defined as $\mathrm{RDR} \leq 50 \%$ and dilatation as $\mathrm{RDR}>150 \%$. The relation of the CMR findings to the combined endpoint of death, stroke, surgery, intervention or escalation of antihypertensive therapy was assessed using Cox analysis.

\section{Results}

A total of 247 patients $(33.0 \pm 12.8$ years, $59.5 \%$ male) were studied. Significant restenosis was present in 9.3\% of patients, while $12.6 \%$ had significant dilatation. A discrete aneurysm at the repair site was observed in $8.5 \%$. Restenosis was more likely in end-end anastomosis repairs and dilatation in patch repairs. Patients with restenosis were at higher risk of the combined endpoint (HR 4.26, 95\%CI:1.83-9.96, $\mathrm{p}=0.001$ ), as were patients with dilatation (HR 2.41, 95\%CI:1.08-5.39, $\mathrm{p}=0.03$ ). Hypertension was a significant predictor of outcome, even after exclusion of the "escalation of antihypertensive treatment" component (HR 3.95, 95\%CI:1.19-13.05, $\mathrm{p}=0.02$ ).

\section{Conclusions}

Restenosis or dilatation at the CoA repair site as assessed by CMR is a predictor of adverse outcome.

\section{Funding}

This study did not have any supporting funding. 


\title{
Author details
}

'CMR Unit, Royal Brompton Hospital, London, UK. ${ }^{2}$ Imperial College, London, UK.

Published: 1 February 2012

\author{
doi:10.1186/1532-429X-14-S1-P116 \\ Cite this article as: Chen et al:: The relation between significant stenosis \\ or dilatation at the repair site and outcome in a contemporary cohort \\ of patients with repaired aortic coarctation as assessed using \\ Cardiovascular Magnetic Resonance. Journal of Cardiovascular Magnetic \\ Resonance 2012 14(Suppl 1):P116.
}

Submit your next manuscript to BioMed Central and take full advantage of:

- Convenient online submission

- Thorough peer review

- No space constraints or color figure charges

- Immediate publication on acceptance

- Inclusion in PubMed, CAS, Scopus and Google Scholar

- Research which is freely available for redistribution

Submit your manuscript at www.biomedcentral.com/submit
C BioMed Central 